\title{
LA INTERACTIVIDAD Y DINAMIZACIÓN EN EL AULA MEDIANTE LA IMPLANTACIÓN DEL DISEÑO DE CASOS PRÁCTICOS JURÍDICOS BASADOS EN SERIES DE ANIMACIÓN
}

\section{INTERACTIVITY AND DYNAMIC IN THE CLASSROOM THROUGH THE IMPLEMENTATION OF DESIGN BASED ON LEGAL CASE STUDY SERIES OF ANIMATION}

\author{
Francisca Ramón Fernández ${ }^{1}$ \\ Vicente Cabedo Mallol ${ }^{2}$ \\ María Emilia Casar Furió ${ }^{3}$ \\ Vicent Giménez Chornet ${ }^{4}$ \\ Juan Vicente Oltra Gutiérrez ${ }^{5}$
}

1. Licenciada y Doctora en Derecho. Profesora titular de Universidad de Derecho civil. Miembro del grupo de innovación docente RETAJUDOCA. Dto Urbanismo. Universitat Politècnica de València, (España). E-mail: frarafer@urb.upv.es

2. Licenciado y Doctor en Derecho. Profesor acreditado titular de Derecho constitucional. Miembro del grupo de innovación docente RETAJUDOCA. Dto. Urbanismo. Universitat Politècnica de València, (España). E-Mail: vicamal@urb.upv.es

3. Licenciada y Doctora en Derecho. Profesora acreditada a titular de Derecho Administrativo Miembro del grupo de innovación docente RETAJUDOCA. Dto. Urbanismo. Universitat Politècnica de València, (España). E-Mail: macafu@urb.upv.es

4. Licenciado y Doctor en Historia. Profesor titular de documentación. Miembro del grupo de innovación docente RETAJUDOCA. Dto. Comunicación Audiovisual, Documentación e Historia del Arte. Universitat Politècnica de València, (España). E-mail: vigicho@har.upv.es

5. Doctor Ingeniero Informático. Profesor titular de Escuela Universitaria. Miembro del grupo de innovación docente RETAJUDOCA. Dto. Organización de Empresa. Universitat Politècnica de València, (España). E-mail: jvoltra@omp.upv.es

Citación sugerida:

Ramón Fernández, F., Cabedo Mallol, V., Casar Furió, M.E., Giménez Chornet, V. y Oltra Gutiérrez, J.V. (2016). La interactividad y dinamización en el aula mediante la implantación del diseño de casos prácticos jurídicos basados en series de animación. 3C TIC: Cuadernos de desarrollo aplicados a las TIC, 5(4), 1-18. DOI: $<$ http://dx.doi.org/10.17993/3ctic.2016.54.1-18/>. 


\section{RESUMEN}

En el presente trabajo se muestran los resultados de la innovación docente desarrollado por los miembros del grupo de innovación docente RETAJUDOCA (Recursos Tecnológicos para el aprendizaje jurídico, la documentación y comunicación audiovisual) tras la concesión del proyecto PIME "Utilización de las series de animación como recurso didáctico en la elaboración de casos" y su aplicación como herramienta para la interactividad y dinamización en la docencia presencial, resultando de especial importancia en titulaciones no técnicas, con la finalidad de conseguir la comprensión del alumno en los conceptos jurídicos.

\section{ABSTRACT}

In this paper the results of teaching innovation developed by the group of teaching innovation RETAJUDOCA (Technology Resources for legal learning, documentation and audiovisual communication) after granting PIME "Using project animated series shown as teaching resource in preparing cases "and its application as a tool for interactivity and dynamism in the classroom teaching, resulting especially important in non-technical degrees, with the aim of achieving student understanding in legal concepts.

\section{PALABRAS CLAVE}

Dinamización, Interactividad, Series de animación, Innovación docente, Casos prácticos jurídicos.

\section{KEYWORDS}

Dynamic, Interactivity, Animation series, Teaching innovation, Legal case studies. 


\section{INTRODUCCION}

La constitución del grupo de innovación docente RETAJUDOCA (Recursos Tecnológicos para el aprendizaje jurídico, la documentación y comunicación audiovisual) en la Universitat Politècnica de València por una serie de profesores de distintas disciplinas jurídicas, documentación y organización de empresas con la finalidad de aplicar las denominadas tecnologías de la información y comunicación (TICS) en las titulaciones ciencias sociales e ingeniería en la citada Universidad ha supuesto un punto de partida para desarrollar una serie de actividades docente, con la finalidad de acercar las nuevas tecnologías y mejorar la comprensión del alumno en los conceptos objeto de estudio. ${ }^{1}$

El desarrollo de la docencia en una Universidad técnica supone un reto para el profesorado de humanidades y ciencias jurídicas y sociales, ya que el perfil del alumnado dista mucho del habitual para el profesorado con formación no técnica.

Para ello, hemos considerado que con las TICs se mejora el aprendizaje y el conocimiento, además de proporcionar al alumnado unas herramientas complementarias para facilitar el proceso cognitivo. ${ }^{2}$ Se tiene que tener en cuenta la evolución de la docencia y la inclusión de los denominados medios audiovisuales y recursos didácticos más allá de la clase magistral. ${ }^{3}$ Con los nuevos planes de estudio derivados del Proceso del Plan Bolonia los papeles o roles de profesor y alumno cambian considerablemente, se pasa de la tradicional clase magistral a la implantación de metodologías activas en el ámbito docente, con la finalidad de que el alumnado sea el auténtico protagonista de su propio aprendizaje. ${ }^{4}$

\footnotetext{
${ }^{1}$ Véase: CANÓS DARÓS, L. y RAMÓN FERNÁNDEZ, F. (2005): «Aplicación de las nuevas tecnologías para el trabajo autónomo del alumno", Actas del XX Simposium Nacional de la Unión Científica Internacional de Radio. URSI 2005, Gandía, págs. 1-4. ISBN: 84-9705-859-3; Depósito Legal: V-3376-2005, y MACEIRAS CASTRO, R., CANCELA CARRAL, A. y GOYANES DE MIGUEL, V. (2010): «Aplicación de Nuevas Tecnologías en la Docencia Universitaria», Formación Universitaria, núm. 1, vol. 3, págs. 21-26. ISSN: 0718-5006. Disponible en: http://www.scielo.cl/pdf/formuniv/v3n1/art04.pdf (Consultado el 3 de noviembre de 2016).

${ }^{2}$ Así: CANÓS DARÓS, L. y RAMÓN FERNÁNDEZ, F. (2007): «La aplicación de las TICs en el aprendizaje de disciplinas jurídicas aplicadas en carreras técnicas en el marco de la convergencia europea», Conocimiento Abierto, Sociedad Libre, Actas digitales del III Congreso ONLINE del Observatorio para la CiberSociedad, Barcelona, págs. 1 a 7. ISBN: 84-611-7675-5; Depósito Legal: B-40053-2007.
}

${ }^{3}$ Cfr. ALONSO GARCÍA, C. M. y GALLEGO GIL, D. J. (1993): Medios audiovisuales y recursos didácticos en el nuevo enfoque de la educación, CETE-ITE, Madrid, págs. 20 y sigs. ISBN: 84-7994-023-9; FERNÁNDEZ MORANTE, Mạ. C. y CEBREIRO LÓPEZ, B. (2003): "La integración de los medios y nuevas tecnologías en los centros y prácticas docentes», Pixel-Bit: Revista de medios y educación, núm. 20, págs. 33 y sigs. Disponible en: http://www.sav.us.es/pixelbit/pixelbit/articulos/n20/n20art/art2003.htm (Consultado el 03 de noviembre de 2016). ISSN: 1133-8482; HERNÁNDEZ REQUENA, S. R. (2008): “El modelo constructivista con las nuevas tecnologías, aplicado en el proceso de aprendizaje», RUSC. Universities and Knowledge Society Journal, núm. 2, vol. 5, págs. 26-35. ISSN: 1698-580X.

${ }^{4}$ Como señalan CANÓS DARÓS, L., RAMÓN FERNÁNDEZ, F. y ALBALADEJO FERNÁNDEZ-SILGADO, M. (2008): «Los roles docentes y discentes ante las nuevas tecnologías y el proceso de convergencia europea», $C D$ Resúmenes comunicaciones $V$ Congreso Iberoamericano de Docencia Universitaria, Edita AIDU. Asociación Iberoamericana de 


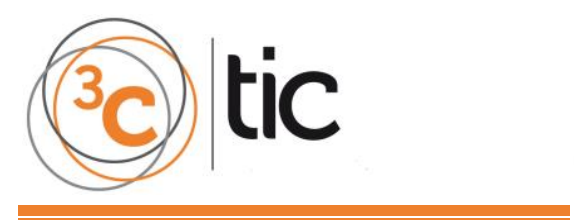

Se parte de una premisa que es la necesidad de incrementar la interactuación y potenciar la dinamización del aula ${ }^{5}$ y conseguirse a través de una innovación metodológica que es el diseño de casos prácticos basados en dibujos animados ${ }^{6}$. Ello supone, por un lado la utilización de las TICs, y por otro, el cambio radical de aplicación de un caso práctico en el aula, ya que tradicionalmente se diseñaban en las disciplinas jurídicas con un soporte predeterminado (un texto ya elaborado) en el que se incluían fragmentos de sentencias o de textos legales, y una serie de preguntas para contestar por parte del alumno.

En el presente trabajo vamos a mostrar la experiencia resultado de la concesión del proyecto PIME "Utilización de las series de animación como recurso didáctico en la elaboración de casos" y su aplicación como herramienta para la interactividad y dinamización en la docencia presencial, en una asignatura optativa del Máster en Ingeniería Aeronáutica en la Universitat Politècnica de València.

No es la primera vez que se utiliza esta metodología en el ámbito de las disciplinas impartidas, ya que también se ha utilizado en alumnos de informática. ${ }^{7}$

Destacar que como resultado de la innovación docente se ha elaborado un repertorio de casos prácticos jurídicos basados en series de animación que se están aplicando a diferentes asignaturas de carácter documental y jurídico dentro de titulaciones técnicas en la Universitat Politècnica de València. ${ }^{8}$

Didáctica Universitaria, Universidad Politécnica de Valencia e Instituto Ciencias de la Educación, Valencia, págs. 388 a 390. ISBN: 978-84-691-8101-0.

5 AEBLI, K. y HUTCHISON, E. (2016): «Classroom Activities to Engage Students and Promote Critical Thinking about Genetic Regulation of Bacterial Quorum Sensing», Journal of Microbiology \& Biology Education, 17(2) DOI 10.1128/jmbe.v17i2.985

${ }^{6}$ Un precedente puede ser el de la Educación Cívica con la serie de Los Simpson, WEHNER, M.; REINKUNZ, S. y FLORY, I. (2009): «Civic Education with The Simpsons», Journal of Social Science Education, 7_8(2_1)86-97.

7 OLTRA GUTIÉRREZ, J. V., RAMÓN FERNÁNDEZ, F., CABEDO MALLOL, V., CASAR FURIÓ, Mạ. E. y GIMÉNEZ CHORNET, V. (2016): «Uso de fragmentos de películas y series como herramientas de innovación docente. Una experiencia con alumnos de informática», INRED. Il Congreso Nacional de Innovación Educativa y Docencia en Red, Universitat de València, págs. 592-602. ISBN: 978-84-9084-541-5. Disponible en http://ocs.editorial.upv.es/index.php/INRED/INRED2016/schedConf/presentations http://ocs.editorial.upv.es/index.php/INRED/INRED2016/paper/viewFile/4430/2000

${ }^{8}$ El repertorio de casos prácticos elaborado se ha publicado en el siguiente manual: CABEDO MALLOL, V., CASAR FURIÓ, Mạ E., GIMÉNEZ CHORNET, V., OLTRA GUTIÉRREZ, J. V. y RAMÓN FERNÁNDEZ, F. (2016): Casos prácticos jurídicos basados en series de animación, Francisca Ramón Fernández (coord.), Tirant lo Blanch, Valencia. ISBN: 978-84-9119-734-8. Depósito Legal: V-1803-2016. 


\section{LA INTERACTIVIDAD Y DINAMIZACIÓN EN EL AULA MEDIANTE LA IMPLANTACIÓN DEL DISEÑO DE CASOS PRÁCTICOS JURÍDICOS BASADOS EN SERIES DE ANIMACIÓN}

Con la finalidad de incrementar la interactividad y la dinamización en el aula se establecieron una serie de estrategias en el ámbito docente con el objetivo de incrementar la participación del alumnado, y que las clases presenciales resultaran menos densas para los mismos.

Teniendo en cuenta el perfil del alumnado y las características de la asignatura que se impartió, vamos a explicar en qué consistió dicha experiencia.

Se trata de un alumnado que presenta las siguientes características:

-Pertenece a una titulación no técnica. Son alumnos del Máster Universitario en Ingeniería Aeronáutica, en la asignatura de Derecho Espacial, que tiene una carga docente de 4.5 créditos (teoría y práctica), y que se imparte en el segundo cuatrimestre.

-No tienen previamente ningún conocimiento jurídico.

-Se trata de alumnos con estancia Erasmus en nuestro país, procedentes de Poitiers. Durante el curso académico 2015-206 se han matriculado 2 alumnos.

-Nivel adecuado de español, aunque con carencias en vocabulario específicamente jurídico.

-Motivación inicial del alumnado, ya que la asignatura es optativa.

Se diseña un caso práctico con la finalidad de ${ }^{9}$ :

a) Mejorar la comprensión del alumno en la lengua.

b) Potenciar el conocimiento de la normativa aplicable a dicho caso práctico.

c) Contextualizar el caso práctico en situaciones reales o ficticias, pero que puedan ocasionar una crítica por parte del alumnado.

Las tres finalidades indicadas se concretaron en los siguientes objetivos:

- Lograr que el alumno comprenda situaciones jurídicas reflejadas en la serie de animación, incrementando su conocimiento y crítica de las mismas.

-Facilitar al alumnado un instrumento audiovisual, a priori, atractivo y muy conocido para que pueda fijar conceptos.

9 Véase más ampliamente: RAMÓN FERNÁNDEZ, F., CABEDO MALLOL, V., CASAR FURIÓ, Mā. E., GIMÉNEZ CHORNET, V. y OLTRA GUTIÉRREZ, J. V. (2016 a): «Diseño de casos prácticos jurídicos basados en series de animación. Una innovación docente con soporte audiovisual», Docencia del Derecho y TIC: innovación y experiencias metodológicas, Ana María Delgado García e Ignasi Beltrán de Heredia Ruiz (coord.), Huygens Editorial, Barcelona, págs. 123-132. ISBN: 978-84-15663-55-3. 
-Incrementar su capacidad crítica, tanto a favor como en contra, a través de argumentos científicos en situaciones no científicas.

-Contextualización de situaciones reales o imaginarias atemporales en las que se reflejen aspectos discutibles relacionados con el ámbito jurídico.

-Potenciar el trabajo colaborativo entre los alumnos, a través de grupos de debate para detección y resolución de problemas, trabajo en equipo, generación espontánea del conocimiento, y su puesta a disposición del resto de participantes, con una exposición pública, en la que se demuestra su habilidad comunicativa, organizativa y de planificación de tareas.

Ello ha supuesto la aplicación de una metodología específica que ha consistido en el diseño de una forma específica de caso práctico, a través del diseño del mismo, el visionado de uno/s episodios de series de animación y unas recomendaciones para el debate por parte del alumnado.

Vamos a explicar, un poco más, el desarrollo de la innovación:

Como hemos indicado en el perfil del alumnado destinatario, eran alumnos Erasmus, con un nivel alto de comprensión del idioma, pero con determinadas carencias en el ámbito jurídico.

La idea era facilitarles el aprendizaje de conceptos jurídicos mediante un sistema no rígido y que les supusiera un aliciente añadido a las actividades a desarrollar en el aula.

Se diseñó un procedimiento a seguir que incluía las siguientes etapas:

a) Elección de la serie de animación que iba a propiciar el incremento del autoaprendizaje, tenía que ser conocida a nivel internacional y no ser excesivamente completa, y que recogiera situaciones explicadas previamente por el profesor en la teoría.

b) Elaboración de una guía a seguir por el alumno para la resolución del caso, previo el visionado del episodio de animación.

c) Disposición por parte del docente de una serie de preguntas para contrastar la interpretación que aporten los alumnos a la resolución del caso práctico.

Para ello, consideramos que sería positivo el visionado de una serie de animación de forma previa a la resolución del caso práctico, con la finalidad de que el alumno participara de forma más activa y se consiguiera la dinamización en el aula, dado que las series de animación contienen una serie de contenidos que nos podían servir para la experiencia docente. ${ }^{10}$

10 RAJADELL I PUIGgRòS, N., PUJOL MAURA, M. A.y VIOLANT HOLZ, V. (2005): «Los dibujos animados como recurso de transmisión de los valores educativos y culturales», Comunicar: Revista científica iberoamericana de comunicación y educación, núm. 25, 2, págs. 1-11. ISSN: 1134-3478. Disponible en: http://www.revistacomunicar.com/verpdf.php?numero=25\&articulo=25-2005-190 (Consultado el 3 de noviembre de 2016). 
Por las características que ofrecían las series de animación, al incorporar conceptos que nos podía resultar de utilidad para la innovación, nos decidimos por elegir una de las más conocidas a nivel internacional, los tebeos de Tintín, que han sido llevados a la pantalla en forma de episodios de dibujos animados. ${ }^{11}$

La doctrina se ha pronunciado expresamente sobre la utilización de recursos audiovisuales valorando de forma positiva su uso. ${ }^{12}$

También se pretendió que el alumno pudiera aplicar la normativa y considerar si las situaciones reflejadas en la serie de animación eran o no conformes a Derecho.

Se decidió que la serie de animación que mejor se ajustaba a la materia de Derecho Espacial eran dos episodios de Tintín, del historietista belga Hergé, en concreto "Objetivo: la luna" (1953) y “Aterrizaje en la luna” (1954).

11 ÍNIIGO JURADO, A. I.: "Las series de animación infantiles. Un producto global que pretende ser educativo», Ficcionando: series de televisión a la española, Belén Puebla Martínez, Elena Carrillo Pascual y Ana Isabel Íñigo Jurado (ed.), Fragua, Madrid, págs. 95-117. ISBN: 978-84-7074-527-0.

12 Así, indica LEÓN CRUZ, P. (2015): «Docere delectando: series, películas y videojuegos como herramientas de innovación docente», Opción, núm. 4, págs. 656-665. ISSN: 1012-1587. Disponible en: http://produccioncientificaluz.org/index.php/opcion/article/view/20576/20486 (Consultado el 4 de noviembre de 2016), que «Esta familiaridad con el manejo de las nuevas tecnologías y el acceso deben ser aprovechadas, siendo para ello necesario identificar la relación existente entre los contenidos tratados en los medios que el alumnado utiliza y los contenidos tratados a lo largo del temario de las asignaturas». Por su parte, CARRACEDO MANZANERA, C. (2009): "Diez ideas para aplicar el cine en el aula», I Congreso de Español como Lengua Extranjera en Asia-Pacífico (CE/LEAP), págs. 229-267. Disponible en: http://cvc.cervantes.es/ensenanza/biblioteca ele/publicaciones centros/pdf/manila 2009/16 aplicaciones 03.p df (Consultado el 4 de noviembre de 2016), precisa que "el visionado de algunas escenas puede apoyar al contenido de nuestras clases y, a la vez, despertar la curiosidad en nuestros estudiantes", y como afirma QUIRANTES SIERRA, A. (2011): "Física de película: una herramienta docente para la enseñanza de Física universitaria usando fragmentos de películas», Revista Eureka sobre enseñanza y divulgación de las ciencias, núm. 3, vol. 8, págs. 334-340. ISSN: 1697-011X. Disponible en: http://rodin.uca.es/xmlui/bitstream/handle/10498/14397/9 Quirantes Sierra 2011.pdf?sequence $=7$

(Consultado el 4 de noviembre de 2016), "el alumno no sabe, pero cree que sabe. Si las ideas previas son generadas en estudios de cine y televisión, en los que no prima necesariamente el rigor, y son asimiladas por personas que carecen de herramientas para el análisis crítico, acabarán conformando un marco de aprendizaje erróneo". 

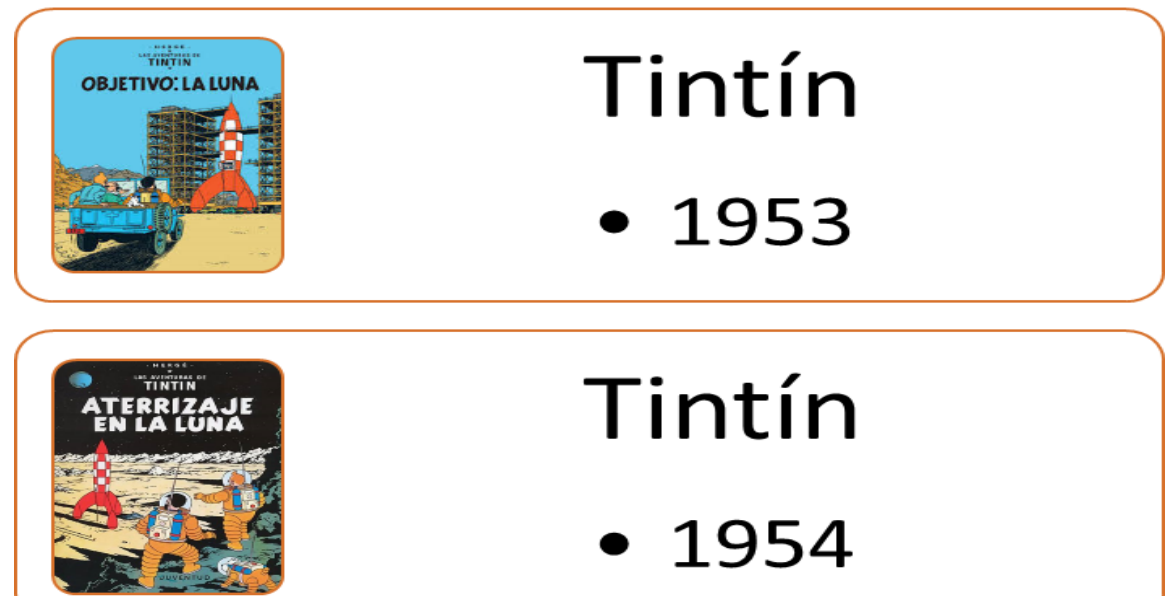

Gráfico 1. Episodios de la serie de animación Tintín utilizados en el diseño del caso práctico. Fuente: Elaboración propia. Imágenes: Portadas volúmenes obra Hergé.

Las razones de utilizar esta serie de animación fueron las siguientes:

1. Serie conocida mundialmente, especialmente los alumnos de Poitiers la conocían previamente.

2. Refleja situaciones críticas en el ámbito del Derecho Espacial, por lo que resultaba idónea para contextualizarla en un caso práctico jurídico.

3. Disponibilidad del visionado de los episodios en francés, lo que facilitó también la comprensión por parte de los alumnos.

4. Amenidad de la serie y corta duración de los episodios, lo que facilitaba la innovación docente.

Se dedican dos clases de la asignatura para el visionado y la resolución del caso práctico. El caso consistía en detectar situaciones previamente estudiadas en clase relacionadas con la legislación ya vista, y considerar si se había aplicado de forma correcta o no la normativa.

La legislación que consideramos adecuada aplicar era la siguiente: 

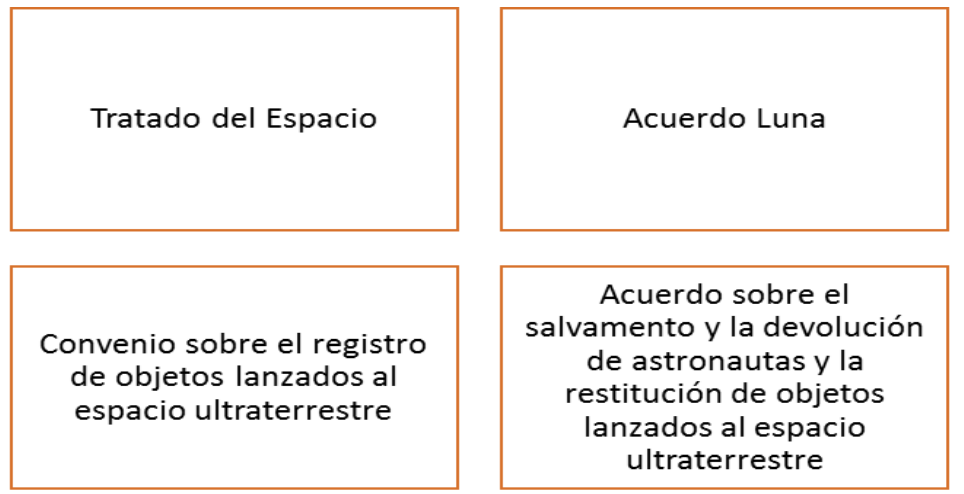

Acuerdo sobre el

salvamento y la devolución

de astronautas y la

restitución de objetos

lanzados al espacio ultraterrestre

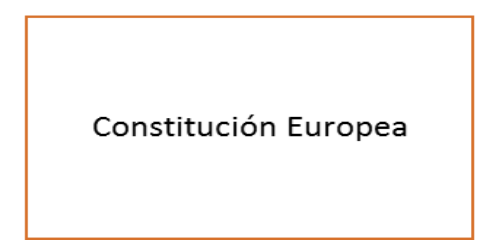

Gráfico 2. Legislación aplicable al caso práctico.

Fuente: Elaboración propia.

Para mejorar la comprensión de la legislación, aunque se explicó previamente en el aula, el alumnado contaba con el soporte audiovisual de los denominados objetos de aprendizaje (OAs), que grabamos los profesores de la Universitat Politècnica de València, dentro del plan de Docencia en Red.

Los OAs que se recomendaron visionar fueron los siguientes:

> Acuerdo sobre salvamento: https://media.upv.es/player/?id=4882c542-a452-a3439ab4-02d42ef975c0\&autoplay=true

$>$ El Tratado del Espacio: https://media.upv.es/player/?id=aed3f890-668a-2841-b4137dca8964525f\&autoplay=true

> Exploración y utilización de la luna: https://media.upv.es/player/?id=ca6e6d26-4793094c-b986-8087c168b734\&autoplay=true

$>$ La investigación científica en la luna: https://media.upv.es/player/?id=5084c8123b1e-e141-bb54-1fb89462241a\&autoplay=true

$>$ Registro español de objetos espaciales: https://media.upv.es/player/?id=5860ad8beaa4-9045-ac4e-608fdbee89d5\&autoplay=true

> Convenio sobre el registro de objetos lanzados al Espacio Ultraterrestre: https://media.upv.es/player/?id=d86cb8c2-9988-2a4e-b6b7-

7b312b57e56c\&autoplay=true

$>$ Acuerdo sobre el salvamento y la devolución de astronautas y la restitución de objetos lanzados al espacio ultraterrestre:

https://media.upv.es/player/?id=4882c542-a452-a343-9ab4-

02d42ef975c0\&autoplay=true

$>$ Declaración de los principios jurídicos que deben regir las actividades de los Estados en la exploración y utilización del espacio ultraterrestre: https://media.upv.es/player/?id=d42c0b8c-ee67-5b45-97c2Obe412295ec0\&autoplay=true 
$>$ Definición de Derecho Espacial: https://media.upv.es/player/?id=cf1156b1-14943642-96e6-4420dfb69890\&autoplay=true

Con el visionado previo de los OAs el alumnado desarrolla una mayor capacidad cognitiva de los conceptos que se explican, ya que están descontextualizados de la asignatura, son reutilizables y tratan de explicar conceptos que se pueden aplicar en diversas titulaciones ${ }^{13}$. En este caso, el alumnado conoce de forma más visual los principales aspectos que regulan la normativa que tiene que aplicar en el caso práctico que realizará posteriormente.

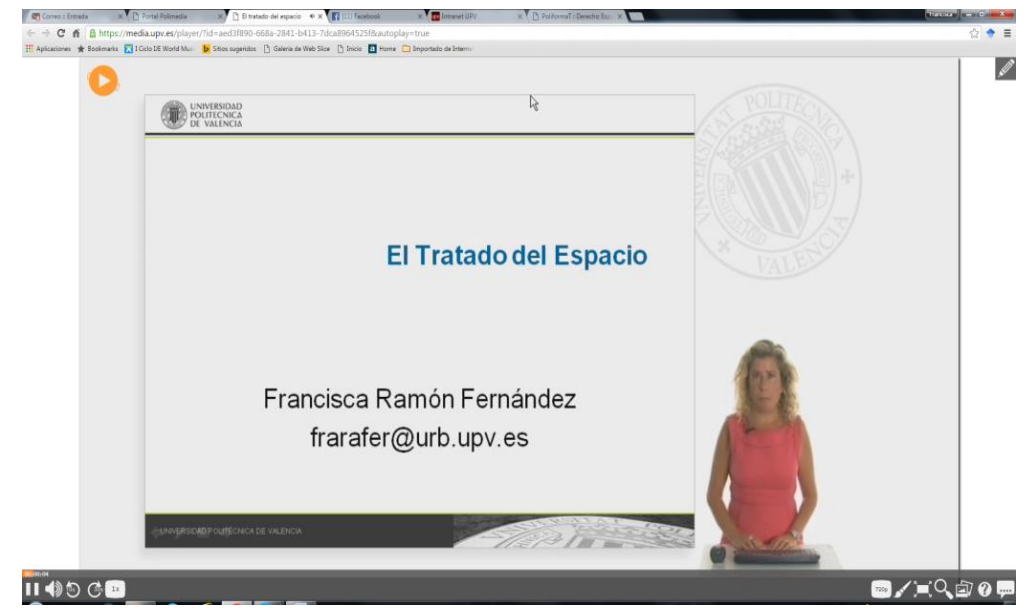

Imagen 1. Ejemplo de OAs. El Tratado del Espacio. Profra. Francisca Ramón Fernández.

Fuente: Universitat Politècnica de València: https://media.upv.es/player/?id=aed3f890-668a-2841b413-7dca8964525f\&autoplay=true

\section{RESULTADOS}

Los resultados que hemos obtenido al implantar durante el presente curso académico 20152016 la innovación docente consistente en el diseño de casos prácticos jurídicos basados en series de animación, en concreto la serie de animación Tintín, con los dos episodios anteriormente indicados, en la titulación del Máster Universitario en Ingeniería Aeronáutica han sido los siguientes:

\footnotetext{
${ }^{13}$ Sobre la utilización de las herramientas online en el ámbito docente, se puede consultar: RAMÓN FERNÁNDEZ, F., CASAR FURIÓ, M. E. y GIMÉNEZ CHORNET, V. (2014): «La aplicación de las TICs a través de la utilización de bases de datos y recursos online en la docencia», Jornadas de innovación educativa y docencia en red (IN-RED 2014), Universitat Politècnica de València, 14 y 15 de julio de 2014, págs. 130-139. ISBN: 978-84-9048-271-1. DOI: http://dx.doi.org/10.4995/INRED.2014 http://riunet.upv.es/handle/10251/40404 http://www.lalibreria.upv.es/portalEd/UpvGEStore/products/p 6183-2-1 http://ocs.editorial.upv.es/index.php/INRED/INRED2014 http://inred2014.blogs.upv.es/
} 
participando de forma activa, y retroalimentándose de los conocimientos a través de su autoaprendizaje autónomo en el aula.

Comprobamos que mejora también la comprensión de conceptos jurídicos, ya que los relacionan con situaciones planteadas en la serie de animación y dicha visualización ayuda a la contextualización del problema.

La contextualización mencionada también ayuda a que el alumnado sea capaz de aplicar una legislación específica, la normativa internacional referente al Derecho Espacial, a las distintas situaciones que se plantean en los episodios de la serie de animación.

Ello facilita que sea capaz de deslindar lo adecuado o no a la norma y las razones que puede esgrimir para una crítica de las situaciones contempladas en la ficción y su aplicación a la realidad jurídica actual.

A ello se suma que el alumnado se atreve más a formular preguntas ante el profesorado y los compañeros que si se realiza una práctica tradicional, sobre el papel y de forma individual.

Se considera, también, que la detección de situaciones jurídicas en los episodios se incrementa porque el alumnado comprende mejor una situación que visualiza, que si se le explican conceptos sin soporte audiovisual.

La valoración del alumnado, a través de la consulta del profesorado, es altamente positiva y prefieren las prácticas soportadas con series de animación que la realización de prácticas con otro tipo de recursos, como por ejemplo, consulta de noticias o análisis de jurisprudencia.

\section{CONCLUSIONES}

La utilización de las TICs en el aula mejoran considerablemente la comprensión del alumno en los conceptos de carácter jurídico.

No podemos olvidar que cualquier innovación con las nuevas tecnologías que se incorpore en el aula lleva un proceso de adaptación, y más aún, cuando estamos utilizando audiovisuales, en este caso concreto, series de dibujos animados. ${ }^{16}$

Es importante conceder la relevancia que se merece al cine, en este caso en su versión animada, ya que constituye una herramienta clave en el proceso de enseñanza-aprendizaje del alumno, más allá de la explicación teórica del profesorado.

\footnotetext{
${ }^{16}$ Como apunta CARRACEDo MANZANERA, C. (2009): «Diez ideas para aplicar el cine en el aula», cit., pág. 229 y sigs., «si bien es cierto que ahora se le reconoce al cine un valor bien merecido y, lo que es más importante, se le va haciendo un hueco en el aula, no siempre fue así. Durante años ha sido relegado al lugar del entretenimiento y desprestigiado por entenderlo como una actividad dentro del ámbito del ocio y fuera del ámbito académico. Lo que considero un grave error. Si en numerosas ocasiones se le reconoce como una actividad de ocio, usemos este argumento a nuestro favor; si forma parte del tiempo libre de nuestros estudiantes, puede ser un factor motivador el usar el cine en nuestra aula».
} 
La interactuación del alumno se incrementa con el visionado de series de animación relacionadas directamente con la asignatura impartida, ya que el alumnado participa de forma más activa en el aula y manifiesta su opinión de una forma más directa.

Se produce una dinamización de la asignatura, ya que permite al profesorado alternar diversos recursos en el aula, con la finalidad de que resulte más didáctica la explicación al alumnado.

Teniendo en cuenta el perfil no jurídico del alumnado, las TICs permiten realizar distintas innovaciones docentes y facilitar la comprensión de los conceptos explicados.

También consideramos que la calidad de la docencia, en busca de la excelencia, se logra de forma más positiva con la incorporación de las TICs. ${ }^{17}$

Las nuevas metodologías en el aula nos proporcionan una mayor interactividad en el alumnado, así como una mayor dinamización de la asignatura impartida. ${ }^{18}$

En el caso de las series de animación como soporte del diseño de casos prácticos se observa el efecto positivo en el aula desde el primer momento. La visualización de un episodio favorece la atención del alumnado, y resulta más atractivo su contextualización, porque le resulta más fácil observar situaciones de carácter jurídico en una trama animada.

Esta dinamización e interactuación en el aula también permite la evaluación de una de las denominadas competencias transversales de la Universitat Politècnica de València, en concreto la CT 6 referente al trabajo en equipo y liderazgo, y en la que el alumno debe «trabajar y liderar equipos de forma efectiva para la consecución de objetivos comunes de un grupo de personas, contribuyendo al desarrollo personal y profesional de los mismos». ${ }^{19}$

17 CANÓS DARÓS, L. y RAMÓN FERNÁNDEZ, F. (2009): «La calidad de la docencia respecto a las nuevas tecnologías y recursos didácticos», Autogestión, cooperación y participación en las Ciencias Sociales, Valencia, págs. 341-352. ISBN: 978-84-613-5902-8.

18 Puede consultarse la experiencia planteada por RAMÓN FERNÁNDEZ, F. (2009): «La implantación de metodologías activas en el aula para la enseñanza y evaluación del Derecho Forestal en la Ingeniería de Montes», Jornada de Innovación Docente en la Escuela Técnica Superior de Ingenieros Agrónomos, Valencia, págs. 163-167. ISBN: 978-84-8363-458-5, y (2014): «La utilización de las TIC en el estudio de casos en la asignatura de legislación patrimonial», Uso de las TIC en la docencia del Derecho: aproximaciones docentes y metodológicas, Ana María Delgado García e Ignasi Beltrán de Heredia Ruiz (Coordinadores), UOC, Huygens Editorial, Barcelona, págs. 197210. ISBN: 978-84-15663-31-7, y especialmente: (2009): «La autonomía en el aprendizaje como metodología activa en Derecho Espacial aplicado a la Ingeniería Aeronáutica», Jornadas de Innovación. Metodologías activas para la formación en competencias y estrategias de evaluación alternativas, Valencia, 2009, págs. 1-15. ISBN: 97884-8363-510-0.

19 RAMÓN FERNÁNDEZ, F., CABEDO MALLOL, V., CASAR FURIÓ, Mạ. E., GIMÉNEZ CHORNET, V. y OLTRA GUTIÉRREZ, J. V. (2016b): «Utilización de las series de animación como recurso didáctico en la elaboración de casos. Análisis de una experiencia en la innovación docente en la Universitat Politècnica de València», INRED. II Congreso Nacional de Innovación Educativa y Docencia en Red, Universitat Politècnica de València, 2016, págs. 920. ISBN: 978-84-9048-541-5 Disponible

en:

http://ocs.editorial.upv.es/index.php/INRED/INRED2016/schedConf/presentations

http://ocs.editorial.upv.es/index.php/INRED/INRED2016/paper/viewFile/4430/2000 (Consultado el 04 de 
Como hemos señalado en los objetivos, la conclusión a la que llegamos respecto al cumplimiento de los mismos es que esta innovación dinamiza e interactúa el aula de forma más favorable, y que con el visionado de la serie de animación y la resolución del caso práctico se incrementa la comprensión del lenguaje jurídico utilizado; así como un aumento más que considerable de la legislación que se ha de aplicar en las diversas situaciones planteadas por el caso, logrando una mayor integración del alumnado en el manejo de conocimientos jurídicos.

Estos resultados positivos han sido también extrapolados en el caso de otras titulaciones, por ejemplo, en alumnos de informática, con lo que el diseño realizado de los casos prácticos basados en series de animación es aplicable no sólo en el ámbito de la aeronáutica, como es el supuesto que hemos desarrollado en este trabajo, sino también en otras disciplinas. ${ }^{20}$

También apuntar, como se desprende de la bibliografía utilizada, que hay experiencias similares referentes a la utilización de audiovisuales en el aula; sin embargo, no hemos encontrado aportaciones sobre el caso que hemos tratado en este trabajo: casos prácticos jurídicos basados en series de animación, por lo que esta experiencia puede resultar de interés para futuros innovadores docentes en las disciplinas jurídicas.

noviembre de 2016) y (2016 c): «La evaluación de la competencia transversal trabajo en equipo y liderazgo utilizando las nuevas tecnologías de la información y comunicación (TICS) en la Universitat Politècnica de València: experiencia en el caso de series de animación», I Congreso Internacional online sobre la educación en el siglo XXI, 10 al 25 de marzo de 2016, págs. 1-9. Disponible en: http://www.eumed.net/eve/educacion.html http://www.eumed.net/eve/esxxi-I/pon/index.htm (Consultado el 04 de noviembre de 2016).

20 OLTRA GUTIÉRREZ, J. V., RAMÓN FERNÁNDEZ, F., CABEDO MALLOL, V., CASAR FURIÓ, Mạ. E. y GIMÉNEZ CHORNET, V. (2016): «Uso de fragmentos de películas y series como herramientas de innovación docente. Una experiencia con alumnos de informática», cit., pág. 598, "Los resultados no pueden ser más satisfactorios. Para poder llegar a esta afirmación aparentemente tan optimista, nos basamos no solo en elementos cualitativos (numerosos comentarios de alumnos, percepción del docente en el aula) sino también en cuantitativos (...). Así, desde el origen de la asignatura, al final de un tema de teoría, se realiza un pequeño test, precedido de un debate. En los grupos donde se ha practicado esta experiencia, el número de test suspendidos (que más que una nota, nos importan en cuanto nos aportan información sobre el nivel de comprensión alcanzado por la clase ante los contenidos teóricos presentados) ha caído en picado, pasando de un 40-50\% a un 10-20\%. No disponemos de la nota total de la asignatura, pues el examen final se realiza en la tercera semana de junio, pero si la tendencia se mantiene, a igualdad de condiciones en el examen final, podemos presuponer un mayor número de aprobados». 


\section{REFERENCIAS BIBLIOGRÁFICAS}

Aebli, K. y Hutchison, E. (2016). Classroom Activities to Engage Students and Promote Critical Thinking about Genetic Regulation of Bacterial Quorum Sensing. Journal of Microbiology \& Biology Education, 17(2) DOI 10.1128/jmbe.v17i2.985

Alonso García, C. M. y Gallego Gil, D. J. (1993). Medios audiovisuales y recursos didácticos en el nuevo enfoque de la educación, CETE-ITE, Madrid. ISBN: 84-7994-023-9.

Cabedo Mallol, V., Casar Furió, Mạ E., Giménez Chornet, V., Oltra Gutiérrez, J. V. y Ramón Fernández, F. (2016): Casos prácticos jurídicos basados en series de animación, Francisca Ramón Fernández (coord.), Tirant lo Blanch, Valencia. ISBN: 978-84-9119734-8. Depósito Legal: V-1803-2016.

Canós Darós, L. y Ramón Fernández, F. (2005). Aplicación de las nuevas tecnologías para el trabajo autónomo del alumno. Actas del XX Simposium Nacional de la Unión Científica Internacional de Radio. URSI 2005, Gandía, págs. 1-4. ISBN: 84-9705-859-3; Depósito Legal: V-3376-2005.

-(2007): La aplicación de las TICs en el aprendizaje de disciplinas jurídicas aplicadas en carreras técnicas en el marco de la convergencia europea. Conocimiento Abierto, Sociedad Libre, Actas digitales del III Congreso ONLINE del Observatorio para la CiberSociedad, Barcelona, págs. 1 a 7. ISBN: 84-611-7675-5; Depósito Legal: B-400532007.

-(2009): La calidad de la docencia respecto a las nuevas tecnologías y recursos didácticos. Autogestión, cooperación y participación en las Ciencias Sociales, Valencia, págs. 341352. ISBN: 978-84-613-5902-8.

Canós Darós, L., Ramón Fernández, F. y Albaladejo Fernández-Silgado, M. (2008). Los roles docentes y discentes ante las nuevas tecnologías y el proceso de convergencia europea. CD Resúmenes comunicaciones $V$ Congreso Iberoamericano de Docencia Universitaria, Edita AIDU. Asociación Iberoamericana de Didáctica Universitaria, Universidad Politécnica de Valencia e Instituto Ciencias de la Educación, Valencia, págs. 388 a 390. ISBN: 978-84-691-8101-0.

Carracedo Manzanera, C. (2009): Diez ideas para aplicar el cine en el aula. I Congreso de Español como Lengua Extranjera en Asia-Pacífico (CE/LEAP), págs. 229-267. Disponible en:

http://cvc.cervantes.es/ensenanza/biblioteca ele/publicaciones centros/pdf/manila 2009/16 aplicaciones 03.pdf (Consultado el 4 de noviembre de 2016).

Fernández Morante, Mạ. C. y Cebreiro López, B. (2003). La integración de los medios y nuevas tecnologías en los centros y prácticas docentes. Pixel-Bit: Revista de medios y educación, núm. 20, págs. 33-42. Disponible en: http://www.sav.us.es/pixelbit/pixelbit/articulos/n20/n20art/art2003.htm (Consultado el 03 de noviembre de 2016). ISSN: 1133-8482. 
Hernández Requena, S. R. (2008). El modelo constructivista con las nuevas tecnologías, aplicado en el proceso de aprendizaje. RUSC. Universities and Knowledge Society Journal, núm. 2, vol. 5, págs. 26-35. ISSN: 1698-580X.

Íñigo Jurado, A. I. (2012). Las series de animación infantiles. Un producto global que pretende ser educativo. Ficcionando: series de televisión a la española, Belén Puebla Martínez, Elena Carrillo Pascual y Ana Isabel íñigo Jurado (ed.), Fragua, Madrid, págs. 95-117. ISBN: 978-84-7074-527-0.

León Cruz, P. (2015). Docere delectando: series, películas y videojuegos como herramientas de innovación docente. Opción, núm. 4, págs. 656-665. ISSN: 1012-1587. Disponible en: http://produccioncientificaluz.org/index.php/opcion/article/view/20576/20486 (Consultado el 4 de noviembre de 2016).

Maceiras Castro, R., Cancela Carral, A. y Goyanes de Miguel, V. (2010). Aplicación de Nuevas Tecnologías en la Docencia Universitaria. Formación Universitaria, núm. 1, vol. 3, págs. 21-26. ISSN: 0718-5006. Disponible en: http://www.scielo.cl/pdf/formuniv/v3n1/art04.pdf (Consultado el 3 de noviembre de 2016).

Oltra Gutiérrez, J. V., Ramón Fernández, F., Cabedo Mallol, V., Casar Furió, Mạ. E. y Giménez Chornet, V. (2016). Uso de fragmentos de películas y series como herramientas de innovación docente. Una experiencia con alumnos de informática. INRED. II Congreso Nacional de Innovación Educativa y Docencia en Red, Universitat de València, págs. 592-602. ISBN: 978-84-9084-541-5. Disponible en http://ocs.editorial.upv.es/index.php/INRED/INRED2016/schedConf/presentations http://ocs.editorial.upv.es/index.php/INRED/INRED2016/paper/viewFile/4430/2000

Quirantes Sierra, A. (2011). Física de película: una herramienta docente para la enseñanza de Física universitaria usando fragmentos de películas. Revista Eureka sobre enseñanza y divulgación de las ciencias, núm. 3, vol. 8, págs. 334-340. ISSN: 1697-011X. Disponible en:

http://rodin.uca.es/xmlui/bitstream/handle/10498/14397/9 Quirantes Sierra 2011.p $\mathrm{df}$ ? sequence $=7$ (Consultado el 4 de noviembre de 2016).

Rajadell I Puiggròs, N., Pujol Maura, M. A.y Violant Holz, V. (2005). Los dibujos animados como recurso de transmisión de los valores educativos y culturales. Comunicar: Revista científica iberoamericana de comunicación y educación, núm. 25, 2, págs. 1-11. ISSN: 1134-3478.

http://www.revistacomunicar.com/verpdf.php?numero=25\&articulo=25-2005-190

(Consultado el 3 de noviembre de 2016).

Ramón Fernández, F. (2009). Diseño de metodologías activas en Derecho Espacial aplicado a la Ingeniería Aeronáutica. Libro Ponencias Decimoséptimo Congreso Universitario de Innovación Educativa en las Enseñanzas Técnicas, Valencia, 15 a 18 de septiembre de 2009, págs. 1-12. ISBN: 978-84-613-4617-2; Depósito Legal: B-36504-2009.

-(2009). La implantación de metodologías activas en el aula para la enseñanza y evaluación del Derecho Forestal en la Ingeniería de Montes. Jornada de Innovación Docente en la 


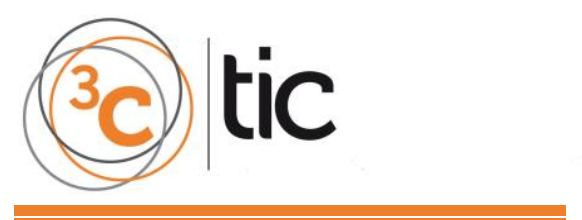

Escuela Técnica Superior de Ingenieros Agrónomos, Valencia, págs. 163-167. ISBN: 97884-8363-458-5.

-(2009). La autonomía en el aprendizaje como metodología activa en Derecho Espacial aplicado a la Ingeniería Aeronáutica. Jornadas de Innovación. Metodologías activas para la formación en competencias y estrategias de evaluación alternativas, Valencia, 2009, págs. 1-15. ISBN: 978-84-8363-510-0.

-(2014). La utilización de las TIC en el estudio de casos en la asignatura de legislación patrimonial. Uso de las TIC en la docencia del Derecho: aproximaciones docentes y metodológicas, Ana María Delgado García e Ignasi Beltrán de Heredia Ruiz (Coordinadores), UOC, Huygens Editorial, Barcelona, págs. 197-210. ISBN: 978-8415663-31-7.

Ramón Fernández, F., Casar Furió, M. E. y Giménez Chornet, V. (2014). La aplicación de las TICs a través de la utilización de bases de datos y recursos online en la docencia. Jornadas de innovación educativa y docencia en red (IN-RED 2014), Universitat Politècnica de València, 14 y 15 de julio de 2014, págs. 130-139. ISBN: 978-84-9048271-1. DOI: http://dx.doi.org/10.4995/INRED.2014 http://riunet.upv.es/handle/10251/40404 http://www.lalibreria.upv.es/portalEd/UpvGEStore/products/p 6183-2-1 http://ocs.editorial.upv.es/index.php/INRED/INRED2014 http://inred2014.blogs.upv.es/

Ramón Fernández, F., Cabedo Mallol, V., Casar Furió, Mạ.E., Giménez Chornet, V. y Oltra Gutiérrez, J.V. (2016 a). Diseño de casos prácticos jurídicos basados en series de animación. Una innovación docente con soporte audiovisual. Docencia del Derecho y TIC: innovación y experiencias metodológicas, Ana María Delgado García e Ignasi Beltrán de Heredia Ruiz (coord.), Huygens Editorial, Barcelona, págs. 123-132. ISBN: 978-84-15663-55-3.

-(2016 b). Utilización de las series de animación como recurso didáctico en la elaboración de casos. Análisis de una experiencia en la innovación docente en la Universitat Politècnica de València. INRED. II Congreso Nacional de Innovación Educativa y Docencia en Red, Universitat Politècnica de València, 2016, págs. 9-20. ISBN: 978-849048-541-5 Disponible en: http://ocs.editorial.upv.es/index.php/INRED/INRED2016/schedConf/presentations http://ocs.editorial.upv.es/index.php/INRED/INRED2016/paper/viewFile/4430/2000 (Consultado el 04 de noviembre de 2016).

-(2016 c). La evaluación de la competencia transversal trabajo en equipo y liderazgo utilizando las nuevas tecnologías de la información y comunicación (TICS) en la Universitat Politècnica de València: experiencia en el caso de series de animación. I Congreso Internacional online sobre la educación en el siglo XXI, 10 al 25 de marzo de 2016, págs. 1-9. Disponible en: http://www.eumed.net/eve/educacion.html http://www.eumed.net/eve/esxxi-I/pon/index.htm (Consultado el 04 de noviembre de 2016). 
Wehner, M.; Reinkunz, S. y Flory, I. (2009). Civic Education with The Simpsons. Journal of Social Science Education, 7_8(2_1)86-97.

\section{AGRADECIMIENTOS}

Trabajo realizado en el marco del Proyecto I+D Excelencia del Ministerio de Economía y Competitividad (DER2015-65810-P), 2016-2018, Convocatoria 2015 -Proyectos I+D- Programa Estatal de Fomento de la Investigación Científica y Técnica de Excelencia. Subprograma Estatal de Generación de Conocimiento, siendo el Investigador Principal el Dr. D. Lorenzo Cotino Hueso, Catedrático acreditado de Derecho constitucional, Universitat de ValènciaEstudi General, del Proyecto MINECO (DER2013-4256R), siendo los Investigadores Principales la Dra. Dá. Luz María Martínez Velencoso, Profesora Titular de Derecho civil, Universitat de València-Estudi General, y el Dr. D. Javier Plaza Penadés, Catedrático de Derecho civil, Universitat de València-Estudi General, y Proyecto "Derecho civil valenciano y europeo" del Programa Prometeo para Grupos de Investigación de Excelencia de la Conselleria de Educación, Cultura y Deporte, GVPROMETEOII2015-014 y del Microcluster "Estudios de Derecho y empresa sobre TICs (Law and business studies on ICT)", dentro del VLC/Campus, Campus de Excelencia Internacional (International Campus of Excellence), coordinado por el Dr. D. Javier Plaza Penadés, Catedrático de Derecho civil, Universitat de València-Estudi General, y Proyectos de Innovación y Mejora Educativa (PIMEs) llevada a cabo en la Universitat Politècnica de València para el curso 2015-2016, del proyecto "Utilización de las series de animación como recurso didáctico en la elaboración de casos", siendo la Investigadora Principal la Dra. Francisca Ramón Fernández, Profesora Titular de Derecho civil. Universitat Politècnica de València. 